\title{
Wolaytta's Quest for Statehood: A Historical Overview and Analysis of Contemporary Quests for Regional Statehood in Ethiopia's Federation
}

\author{
Tensay Hailu \\ Department of Social Anthropology, Lecturer at Wolaita Sodo University, Sodo, Ethiopia \\ Email address: \\ tensayhailu@wsu.edu.et \\ To cite this article: \\ Tensay Hailu. Wolaytta's Quest for Statehood: A Historical Overview and Analysis of Contemporary Quests for Regional Statehood in \\ Ethiopia's Federation. Social Sciences. Vol. 8, No. 4, 2019, pp. 198-205. doi: 10.11648/j.ss.20190804.18
}

Received: June 1, 2019; Accepted: July 8, 2019; Published: September 3, 2019

\begin{abstract}
This paper tries to shed a light on the history of Wolaytta that evolved from huge kingdom to the recent small province called Zone. Here the researcher presents insights on the previous glories of Wolaytta kingdoms and the retreat of the kingdom's political and economic hegemonies. The paper also tries to look at the contemporary quest of independent regional state of Wolaytaa. Issues that have been raised above are qualitatively analysed after extensive data collection through interview, group discussion and formal and informal discussions with elderly people who are aware of the history of Wolaytta. The finding of this research shows that the people of Wolaytta had very strong centralized political system since $13^{\text {th }}$ century. Basically two dynasties under the name called Wolaita-Mala and Wolaytta Tigre had ruled the vast territory of Wolaytta up until late $20^{\text {th }}$ century. The kingdom was also known in expanding to the reset of the country up until the then notorious northern Ethiopia up until Gojjam, Amhara. The state was also defended its territory from the expansionist Minilik II and finally incorporated to the Ethiopian kingdom after a bloody war. Since their incorporation, Wolaytta had faced a century of retreat both economically and politically. With the change of leader in the ruling part EPRDF, a new wave of change has been observed that finally led the resurgence of Wolaita's greatness and the quest for independent regional statehood under the Ethiopia federation.
\end{abstract}

Keywords: Wolaytta, Statehood, Historical Development

\section{Introduction}

Following the Southern Nations, Nationalities and Peoples' (SNNP) State Council's decision to approve a request for a referendum on Sidama statehood, tensions have started to pour throughout the entire region of southern Ethiopia. As a result of the approval for a referendum on sidama statehood, historically independent and demographically strong nations have started to forward their demand of statehood. In this regard, On Nov. 26, 2018, the Gurage people's Zone Council voted to demand Gurage state for the SNNP state council. Two weeks prior, Wolayta Zone affirmed a statehood request, Kaffa Zone also approved a demand on Nov. 15, shortly after locals protested over a suggestion that coffee originated from Jimma.

The big danger is that the moves increased identity, border and resource-related conflicts that have plagued the 21 million strong Southern Nations, which has around 50 recognized indigenous groups. There has historically been plenty of jostling for more administrative autonomy in SNNP as that generally gives local elites greater control over spending and appointments [1].

While the statehood ambitions are legitimate the South is a fragile region of interwoven ethnic groups with unclear territorial borders which have been the prime cause of interethnic conflicts. In June 2018, Wolaytta people were killed in Hawassa, the capital of southern nation and nationalities region, and violence flared between the Gurage and Kebena groups around Welkite. In three other locations in SNNP, 16 people were killed in July and August 2018, according to the Ethiopian Human Rights Council (HRCO). In August of the same year, violence in Tepi that killed five people stems from the Sheko people's desire for autonomy, HRCO said. There was more fatal violence in Gurage the same month [1]. 


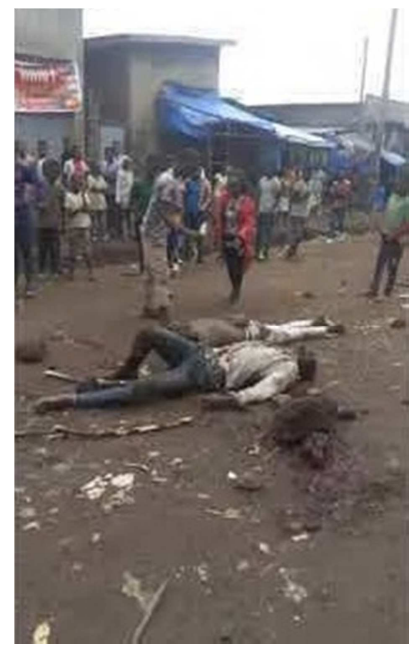

Figure 1. The tragedy, mass killing and torturing in and around Hawassa in 2018. As it is observed in the picture, ethnic Wolaaytta were killed and brutally burned in front of fellow human being.

The killing of Wolaytta people in Hawassa even intensified their demand for independent statehood in Ethiopia's federation. The mass killing further triggered different politically motivated groups working on human right and related political issues of Wolaytta people. The Wolayta Committee for Human Rights (WCHR) and Wolaytta's youth movement, famously called 'Yalaga' are some of those organized movements pushing hard for the independence of Wolaytta people from the pseudo state that defy the logic how the current states in Ethiopia are organized ${ }^{1}$.

Now, who are Wolaytta people? How did their history can be narrated? How their demand for such an independent statehood did evolve? What are the historical and constitutional grounds that validate Wolaytta's demand for independent statehood in Ethiopia? This article will have the answer for all these questions.

\section{Methods and Methodology}

\subsection{Methodology}

Since the paper is historical manuscript, the researcher used qualitative data and the analysis was made qualitatively. Here different open ended questions both for the key informant interview and focus group discussion was prepared and selected informants of this research are approached to provide information accordingly.

\subsection{Methods of Data Collection Techniques}

Historical researches mostly use written documents both in the form of published books and archives. Because of this reason, the researcher has tried to use as many literatures as possible while primary sources of data are also incorporated as it is mentioned below.

1 All except one state in Ethiopia are organized based on their ethnic background. However, the state called Southern Nations and Nationalities Regional State is organized based on geographical proximities of 50 nations in the region.

\subsubsection{Primary Source of Data}

Key Informant Interview: there were five key informants who are selected based on their knowledge of history, culture, language and the current socio-political conditions of Wolaytta people. The researcher used snowball method to select those key informants. Moreover, these five key informants have been approached at least twice during the data collection stage.

Observation: This technique helped the researcher to get information that is left during formal interview sessions with informants. Hence, observation selected to be one of our data gathering techniques just to grasp information about the unsaid things during the interview and informal conversation sessions.

In this regard, observational checklist was prepared before the actual fieldwork activities in the study area. During data gathering process in the field, the researcher observed historical places of Wolaytta, heritages of Wolaytta including prehistoric Eco facts and artifacts, mass demonstrations called by Wolaytta youths up on their struggle to make Wolaytta one of the regional states of the country. The researcher took notes during his observation and used those notes during the writing up of this manuscript.

Informal Conversation: since topics like this research are politically sensitive, people always resist to participant as an informant especially in a formal interview sessions. Moreover, some informants preferred informal conversations than the one which is formally prepared because they suspect that their view could be used for surveillance. Therefore, Informal conversations have been used to collect data from different government and none governmental, the youth also called Yalaga and activist.

\subsubsection{Secondary Sources of Data}

Published, unpublished and online literatures are used to have some sort of data on different key concepts, background of the study area and key findings on similar topics. Moreover, these types of data were used to have pertinent backgrounds, to make comparison and to aid empirical data gathered from the field. In doing so, the researcher managed to use different books, researches, non-Wikipedia on line sources, archives and institutional and governmental documents. On top of that the constitution of Ethiopia was also used to evaluate basic demands of Wolaytta people with respect to the country's constitution.

\section{Data Analysis and Discussion}

\subsection{Historical Background of Wolaytta People}

The history of Wolaytta dates back to the pre historic period [2]. Some archaeological excavations have shown that the people of Wolaytta used to live in some major caves before thousands of years. Findings of the group of archaeologists under the leadership of professor Stevie further claimed that the people were used to carve caves around rivers and cultivate crops in order to survive hard times. As evidence to their claim, the group have presented 
different grains and lithic technologies that have been found from their excavation [12].

Moreover, evidences of the historic period have shown that the people of Wolaytta had been living in a mountainous area called Kindo Didaye [3]. It is widely believed that ancient Wolaytta had fled to this mountainous region for presumably two reasons. The first assumption is that some natural disasters forced them to relocate their residence. Secondly, the need to have arable land for cultivation of food could be the reason. Despite the controversy of how, when and why ancient Wolaytta relocated their residence to Kindo Didaye, we can surely claim that the people of Wolaytta had managed to evolve to a greater social, political and economic status ever since their time in kindo didaye [4].

Ever since their time in Kindo Didaye, Wolaita have stated cultivation of massive lands and production of different crops both for consumption and for market exchange. Gradually they have introduced their own currency called Merchuwa. The innovation of currency had undoubtedly become one of the turning points for the evolution of Wolaytta people to a greater kingdom. It has led them to boss trade relation with neighbouring people, become the centre and hub of commercial exchanges and the leader of socio-political issue of people in and around the state of Wolaytta. Demographic and economic growth of Wolaytta further intensified and led for the creation of more centralized political administration which is led my Kawo, means king [3].

There were series of kings under two major dynasties ${ }^{2}$. These are the Wolaytta Mala and the Wolaytta Tigre Dynasty. The former is believed to be the founder of the first centralized state of Wolaytta. This clam in validated by the non-prevalence of centralized state of Wolaytta before this dynasty. One of the famous elder of Wolaytta who is referred as the main source of Wolaytta history Eyasu Gejebo says:

There was no centralized administration before Wolaytta Mala. Before the rise of such centralized administration system, Wolaytta was ruled by the council of elders who rule their respective constituency. When there is conflict and aggression from outsiders, the council meet and decide on how to overcome the problem together and mobilize their respective constituency for the objectives set by the elders.

Interview: Eyasu Gejebo.

Wolaytta Mala therefore has established the first centralized state under the leadership of $\mathrm{Kawo}^{3}$ Mero and lasted up until Kawo Halala. After Kawo Halala, Wolaytta mala was replaced by Arujiya dynasty under the first kingship of Kawo Gadao. However, the reign of Arujia dynasty did not last long but overthrown by the former dynasty, Wolaytta Mala dynasty [12]. This is the second time

2 Arujia is arguably the third dynasty that is taught to rule Wolaytta for some time in the $15^{\text {th }}$ century. However, elders claim as Arujiya rulers as non-indigenous. They further claim that Arujia rulers had caused massive devastation to the economy and caused huge mal-administration problem in the region. Arujia is finally overthrown by Wolaytta Mala dynasty, a dynasty who was ruling the region before the coming in to power of the outgoing dynasty, Arujia.

3 Wolaytta people call their king as Kawo, a word synonymous with the English word King. when Wolaytta Mala comes to the power and rule the entire territory of Wolaytta for almost four hundred years. During this time Wolaytta Mala had been strengthened and expanded Wolaytta's territory further to all direction. Especially during the leadership of King Motolomy, Wolaytta managed to expand as far as the northern part of shewa provinces and Gojam. The final dynasty was named as Wolaytta Tigre Dynasty ${ }^{4}$. The Tigre dynasty lasted up until their last king Tona Gaga was invaded and surrendered after series of war and skirmish with the then Ethiopian King Minilik II [5].

Wolaytta's self-administered kingdom was abolished after the severe war that has led the death of thousands of people, devastation of the economy and overthrow of their king by Ethiopia king. Wolaytta surrendered and treated as one of the province of the entire Ethiopia's empire. Rulers were appointed from the centre and rule Wolaytta and its surrounding for long period of time [5].

\subsection{Wolaytta Dynasties}

Since $12 \mathrm{C}$ A. D. Wolaytta people had centralized political system which has state like structure of modern days. Here, different Wolaytta kings by the name Kawo ${ }^{5}$ have been ruling the entire Wolaytta people with their own peculiar government structure and administrative systems. There were three dynasties from which the kings were coming from and coroneted to rule Wolaytta [3].

The First Kingdom was named as Wolaytta Malla dynasty that is believed to rule in between $12^{\text {th }}$ century to the end of $14^{\text {th }}$ century. The first king in the modern administrative history of Wolaytta is called Kawo 6 King Mero and the last king of the Malla dynasty was named as Kawo Hallala. After King Hallala, a new dynasty was established to rule the people of Wolaytta, this dynasty was known as Arujiya dynasty where many elders of Wolaytta believe them to be a non Wolaytta dynasty [3].

Kings of this dynasty were portrayed as harsh very rude and killers. According to elders, there were 12 kings under this dynasty who ruled Wolaytta. Unfortunately, the end of the dynasty is not clearly known ${ }^{7}$. However, some elders claim the Arujiya who are arguably considered to be nonWolaytta rulers, were lost a battle to the Malla dynasty who ruled Wolaytta before the rise of Arujiya dynasty. The revival of the Malla dynasty and downfall of Arujiya is famously known by the Wolaytta people as the restoration of Malla dynasty.

The restoration of Malla Dynasty was led by their first Kawo Sene and ended with Kawo Leche. It is claimed that 15 kings of Malla dynasty ruled Wolaytta. Then after the last Malla dynasty kawo Leche, a new dynasty called Wolaytta

\footnotetext{
4 Some Wolaytta elders describe this dynasty as it was established by Tigre descendants whose centre is situated in the northern part of the country, today's Tigray regional state. These people are believed to come for either for trade or as part of the expansion of king Made Tsion of Abyssinia in the $14^{\text {th }}$ century.

5 Kawo is a name that Wolaytta used to call their king. The word has equivalent meaning with that of king in English vocabulary.

6 Wolaytta people call their king as Kawo. Kawo is local word meaning king.

7 Informant Eyasu Gejebo
} 
Tigre took the power with their first king called Kawo Michaela or Shume. The Tigre dynasty ruled Wolaytta for centuries and end up after a bloody war with King Minilik of Ethiopia, who ruled the imperial government of Ethiopia in between 1889 to $1913^{8}$. The famous king of Wolaytta Tona has fought to defend his territory from Minilik's force who wanted to conquer Wolaytta land and exploite the resource. Unfortunately after series of war and battled fields, Kawo Tona surrendered to Minilik's force as Wolaytta become part and parcel of the notorious empire of Minilik [6].

What makes all the dynasties very similar and common is that they all have their own very strong security system both in the palace and in major towns of their kingdoms, defence force equipped with locally made armaments, very sophisticated political and administrative structures, economic regulation system, currency and justice system with profound judiciary and arbitrary system. According to Zebdios Chama', Wolaytta's well-known and well informed elder, Wolaytta Kings had government workers who serve the government and public in providing services like security, revenue collection, justice provision and trade regulation.

Kings inherit their power for their relatives including among from their children called the Bushasha, or legal hairs of the king. Normally kings are replaced by the Bushasha. They overtake the throne whenever the king is dead or else the king is severely ill and cannot lead the people with his health status. The death of kings however remain top secret of the palace and it will never be announced up until another king is replaced [14].

\subsection{The War with Menilik II}

The Ethiopian empire, having its centre in Shoa, expanded its territory southward at the end of the nineteenth century. Before their expedition to Wolaytta, the force of Minilik fought and incorporated the Amhara's ruler Teklehaymanot in 1874, the so called Shoa Oromo people in between 18671880's, kingdoms of Ghibe after successive wars in 1879 and Arsi Oromo similarly in 1879. Before his expedition to Wolaytta, Minilik fought a bloody war with Hararghe Oromo people at the battle of Chelenko in 1887 [7].

Wolaita was incorporated in 1894, after "one of the bloodiest campaigns of the whole process of expansion." This powerful kingdom opposed a determined resistance to Ethiopian armies. As all the other kingdoms that resisted, Wolaytta was treated harshly. This is because the already existing strong kingdom in Wolaytta had fought for its existence and managed to win Minilik's force repeatedly. The fact that Wolaytta force won Minilik's force did not impress the strangers, it rather make them to be more brutal [14].

They have used advanced artilleries that they bought from European nations that have had diplomatic relation with the

8 Informant Tadese Anjeja, one of the known Wolaytta elders.

9 Zebdiwos is one of my key informants and very informed person about the history and culture of Wolaytta people. He is writing his own book on the history of Wolaytta. He is also one of the very proactive people who have been fighting for the preservation of Wolaytta heritage, culture and history. then kingdom of Ethiopia. They have also got some arsenals as gifts in different times when diplomatic mission was visiting the emperor. Apart from advantage in war armaments, Minilik's force was relatively a trained one that was taking command from the centre and lead by war leaders who have strategies of war. Moreover, the strangers were well aware of the traditional equipment that Kawo Tona was using especially after their successive defeats in different battle fields they fought with Wolaytta army in early 1894 $[5]$.

Despite the abovementioned advantages in artillery and military preparation, Wolaytta had fought with full courage and determination to protect their kingdom. In doing so they have used traditional methods of attacking and defending mechanisms that have been used $b$ force second to none in the history of warfare. For instance, honey bees, different spears both in size and shapes, spears spoiled in their edge were used to attack invader. They were digging trenches and small hills are also used to observe and attack enemies. The Wolaytta army was also used a shield that is locally made. What makes Wolaytta army more different is that they were using a locally prepared cloth which is very huge and in terms of its thickness. Special close was also worn to the army from any bullet or spears directed to them ${ }^{10}$.

After a severe bloodshed and loos of life from both sides, Minilik's force had managed to win the war at last. Despite the loose in the battle field which is now located around Delbo, Wolaytta people still remember the reason why their army was lost the war. My informant Zeleke Bololo said ${ }^{11}$.

Wolaytta lost the war not because the enemy had got it with fight but because the enemy had got the weak leak of our army using paid natives who worked for the enemy. It is well know that Wolaytta people love their family more than anything. Hence, Minilik's force was told to burn Wolaytta's house and threaten the life ordinary citizens who are believed to be relatives of the army. The idea was to dismantle Wolaytta's force while they are fighting half-hearted to save their family.

Informant: Zeleke Bololo.

The information was right and when they burn the villages that Wolaytta people used to live, the army started to disperse to their respective village to save their family from the allegedly burning villages. Due to this reason as Minilik's force managed to outnumber Kawo Tona's army and eventually win the war.

Shoa's victory was followed by massacre, plunder, and massive enslavement. Land was distributed to the soldiers of the victorious army, who were granted the right to levy taxes and exploit the labour force of the peasants. The conquerors quickly assimilated local families to their rule. The new ruling class then included northern settlers and co-opted Wolaytta. A harsh system of domination and exploitation was imposed on the vanquished peasant population [14]. Haylä

10 Interview with Tadesse Anjeja, Bezabih Baza, and Esayas Eshacho tends to inform the above mentioned history of Wolaytta during Minilik II's invasion.

11 We had an interview session with Zeleke Bololo in Wolaita Sodo town in February 28, 2019. 
Səllase's administrative reforms after 1941 did not significantly affect the local power relations. Neither the gradual replacement of soldiers by civil servants nor the privatization of land improved the condition of the peasantry.

\subsection{The Period of Abatement}

\subsubsection{Wolaytta After Minilik's Invasion}

After the invasion of Minilik's force, the town of Delbo which was used as the main metropolis and government centre of Wolaytta was replaced by Sodo, a town which is used as the sitting place of the zone ${ }^{12}$ administration currently. The town of Sodo was used as the centre of Minilik's administration where war lords and their relatives were used to live. Leaders were appointed by the king directly and used to make their residence in the town. Despite their victory in the last battle, Minilik's force was failed to maintain peace and order in the region for long period. Due to this reason the strangers were focused on maintaining peace in the entire region [8].

Wolaytta was never managed to reach the extent of economic and political highness ever since their incorporation to Ethiopia. They were led by appointed leader from the centre whose knowledge about the area is impaired. On top of that, the rulers are only coming to Wolaytta with only one thing in their mind, exploiting the resources that Wolaytta has and transfer it to the centre. Some of the rulers are even assigned to the area as a demotion since the area is considered to be remote and their presence in the so called remote area pose little threat to the kingdom in the centre.

The cumulative of the abovementioned reasons had caused the immediate decline of Wolaytta's as a state and impoverished the entire state for a very long period of time. Now, the imperial regime was eliminated but Wolaytta's socio economic decline continues even harder this time. Here, the researcher has presented to chronologically dichotomized periods that shows how Wolaytta people were treated in two ideologically different regimes [14].

\subsubsection{Wolaytta During the Period of Derg ${ }^{13}$}

Derg was famously known for its socialist political ideology which was the fashion of the then eastern blocks including overwhelming majority of southern American countries. Culture and ethnicity was considered as a dividing device that the then Ethiopia treated it harshly. In this particular time the entire nations and nationalities of the country were forced to forget their ethnic identity while they are continuously preached about the statehood of greater Ethiopia [9].

Just like the imperial regime, Durg had preferred to send governors from the centre, Addis Ababa. Most of the leaders were fanatic socialists who are blueprinted version of the

12 Zone administration is the second largest administrative segment of the country next to regional administration. Hence Wolaita zone is one of the zones under the southern nations and nationalities regional state comprising more than 13 zones.

13 Derg is the military administration that overthrows the imperial regime in Ethiopia and established a socialist state for 17 years in Ethiopia. centre. Mass killings, torture and harassments were common in Wolaytta as it was in the rest of the country. Especially those who are suspected to be members of EPRP (Ethiopian People Revolutionary Party) were brutally killed in major public spaces of Sodo town and in front of massive public gatherings. Due to such mass killings, a generation had been lost. The scarce of such killings remain bold still today due to two reasons.

The first reason is that those who are believed to be members of EPRP and killed by the then government were literates who managed to join the then prestigious university of Addis Ababa. According to informants, they could have transformed Wolaytta if they were in life. The second reason was that Wolaytta's resistance to their oppressors seems slowed down due to the absence of those killed Wolaytta people, absence of genuine movement above all due to the trauma that was created due to horrifying mass killings.

\subsubsection{Wolaytta Under the Incumbent Authority}

Immediately after the coming in to power of EPRDF, The fate of the southern people had become the source of controversy both among different ethnic groups of the region and even among elites of the country in general. There was a supposed preliminary discussion before the decision to assimilate more than forty nations and nationalities in the region. Sadly those who represented the region and take part in that discussion were members of the ruling part who care for nothing than appeasing their masters. The decision to mould the entire nations and nationalities of the region in to one state called southern nations was not feared to be brutal as it is gradually observed.

Especially for those that have long political administration history, massive and sovereign territory and self-sufficient economy like Wolaytta, the decision was considered as an insult to their history. One thing that must be admitted was that the people of Wolaytta were granted self-administration after a longer period of time. But Wolaytta still remain under the leadership of regional leaders whose inherent care was for their constituents.

The decision was still fallacious when we observe how regional states have been organized elsewhere in the country. Here no other regional state in the country was organized based on their geographical location but only based on their ethnic identity, which the writer of this paper believes as the cause for the recurrent political instability in the country. By tracing the prejudiced administrative systems of the imperial regime towards the southern people, some still argue that the southern part of the country is still mistreated by the central government of the current federation.

\subsection{Wolaytta's Quest for Regional Statehood}

Wolaytta is now demanding the highest possible administrative structure, a regional state. The demand is supported by a strong and deep rooted movement led by the Wolaytta youths famously known as Yalaga. The people of Wolaytta severely demand socio-economic and political reforms. The reforms that the people are demanding however 
cannot be answered by a state which is organized and ruled by others. Wolaytta needs to go to the bottom of their problems themselves. In order to do so, they need the political and administrative power that gives them to bargain with the central government.

Wolaytta's quest for statehood can be justified in three ways. Here, the writer has presented those justifications below.

\subsubsection{Historical Claims}

Historically, as we have seen earlier Wolaytta was a strong nation which was administered by strong centralized administrative system. As it is repeatedly confirmed by both local and foreign writers, Wolaytta remain a strong hold of dynasties that are emerged from Wolaytta itself. In this regard, big centres like Halala from the south western, Damota from the centre, Delbo in the north had been used to be the administrative and political centre of Wolaytta nation.

The state had its own modern administrative structure where power is shared among government officials and balance of power is checked. For instance there are different government advisors called zore mochenata, Ginda a position left for those who advise the king regarding the economy of the nation, Meaga, war leaders. Here the nation had its own military force equipped with traditional armaments that are produced locally. The military had been trained, organized and commanded by a central unit and administrative wings that are dedicated only for this cause.

The people of Wolaytta in general and the then state in particular had their own currency by which the people exchange goods and service and the government collect its revenue from the people. The currency was called Merchuwa, produced by local artisans. The technique of its production and materials that are used for its production still remain mysterious. When Wolaytta managed to exchange goods and service via their own currency, barter was the only way of exchanging in the entire Ethiopia except in the northern section of the country.

However, with the coming of the imperial power, the decline of Wolaytta's nation had become imminent. The history, the culture and language of the people was systematically forced to be abandoned. The people of Wolaytta therefore are subsumed under the Ethiopian empire by losing its indigenous political administrative power and the strong state ruled by its own kingdoms.

Historically therefore, Wolaytta was used to be a strong state having its own strong economy, defined and well protected territory, independent administrative structures, social and cultural identities that distinguish Wolaytta from its neighbouring people [6]. Hence, the contemporary quest for regional statehood is not a new demand rather it must be considered as a quest of retaining the independent nation of Wolaytta back. On top of that the current movements that are claiming the statehood of Wolaytta should be considered as resurgence movements that demand the old Wolaytta back.

\subsubsection{Constitutional Grounds Validating the Quest}

Wolaytta's quest for self-administration is constitutional and it can be validated by tracing the current constitution of Ethiopia. The constitution of the country is not against any demand of all nations and nationalities who demand selfadministration in the first place. It has procedural ups and down but not complete overrule. Here the writer has presented constitutional grounds that are validating Wolaytta's quest for statehood.

Article 30 sub article 1 talk about 'the right to petition' and demand whatever certain nation wanted to be done for them. The article further mentions the responsibility of administrators and government organ to act accordingly to the demand of the people that have signed petition in request of their demand. In this regard the people of Wolaytta have signed a petition and demanded rulers to act up on their demand. Did their quest have the appropriate answer accordingly? History will give us the answer [10].

Article 39 sub article 1 talks about 'the right of nations to determine their fate'. Here the article is granting nations and nationalities that they have the right to call for selfadministration whenever they believe is the right time. Now Wolaytta is not administered by themselves, there is a superficial state which is not organized based one ethnicity as it is done everywhere else in the country or following the demand of the people of the region [10].

Article 39 sub article 3 mentions the unrestricted right of the people to administer themselves. The full article reads:

Every nation, nationality or people of Ethiopia shall have the unrestricted right to administer itself; and this shall include the right to establish government institutions within the territory it inhabits and the right to fair representation in the federal and state government.

FDRE Constitution of Ethiopia, Article 39 Sub article 3.

The article clearly put the unrestricted right of nations to administer themselves and have representation in the federation. Here the writer believes that Wolaytta people are unders a huge restriction of self-administration that SNNPR put on the shoulder of the people of Wolaytta.

The other Article that validate Wolaytta's demand is the black and white statement in the Article 39 sub article 5 that reads:

"nationalities shall have, on the basis of the free choice of their people, the right to organise on a large territory a self-administrative structure for running their internal affairs and establish governmental institutions for common self-administration."

FDRE Constitution of Ethiopia, Article 39.

Here the article mentioned that the people shall have the right to establish a regional self-administrative unit on the basis of their demand and the procedure that they must fulfil. The procedures are mentioned under article 30 sub article 5. Currently the demand to establish regional selfadministrative unit is approved in zonal level but got a deaf ear in regional level, where such treatment is expected to happen in the first place [10].

Article 39 sub articles 7 says that by the virtue of an unjust distribution of social wealth or an unfair distribution of the product of development, a sector of population which has 
obtained recognition as a nation can raise the demand for secession, the causes for which the demand was made shall be made to find solution. Here unfair distribution of wealth and allocation of resources was reported by the people of Wolaytta nearly for a century. However did the central government find any solution for it? Never, and now the people has clearly come in to a conclusion that their unfair treatment and maladministration under strangers only come in to an end whenever their quest for statehood is answered [10].

\subsubsection{The Demand of the Mass}

We have scanty of information about the leadership after the death of Minilik up until the coronation of Teferi Mekonin as the king of Ethiopia. Despite the lack of written sources about it, informants clam that there was no shift of administration system even after the death of Minilik II. Still, administrators were appointed to lead Wolaytta and its surrounding. However a little shift has been made after the invasion of Italy in 1936. After the 5 years Italian occupation Ethiopia was divided in to 12 Awraja or administrative province [5].

Just after a while the Awraja was renamed as Teqlay Gizat that is still a province but a province which is relatively bigger than that of Awraja. The number of Teqlay Gizat was never been constant as it is increasing and decreasing time and again. For instance the number of Teqlay Gizat was once reached 14. The 14 Teqlay Gizat was further divided in to distinct Awraja. The Awrajas were also divided into Weredas and Woredas as well were divided into Mikitil Woreda [11].

Wolaytta was mentioned under the Sidamo Teqlay Gizat and was one of the Awrajas of the country in mid- $20^{\text {th }}$ century. The decision was not popular by the people of Wolaytta. It was rather considered as a disgrace for the people who had been a country by themselves and a peple who had their own modern administrative system led by kings. Even though mass revolt was not observed openly, there was a wider discontent and continuous dialogue to make Wolaytta as one of the Teqlay Gizat between elders of Wolaytta and the then leadership of the region [11].

The people of Wolaytta made it clear that they want to form their own regional state time and again. Tracing history, we can find Wolaytta elders who stood firm towards the decision of Haile Selassie's government that organized Wolaytta as sub region. The elders' quest was not violent so that it can be herd and publicized to affect the then administrative units of the government ${ }^{14}$.

According to informants, there were unorganized raids against rulers who appointed to rule Wolaytta since the imperial regimes. Because of such raids targeting only non Wolaytta rulers, many appointed men are less interested to come to Wolaytta. Dejach WeldeSemeat for instance was one of the rulers who initially refused to come to Wolaytta because of the people's strong demand of self-administration and resurgence of the old dynasties. According to informants the people were really looking to be administered by their own people since the imperial regimes.

In 1941 Ethiopia introduced new administrative regions once again. The regions were named as Awraja Gizat, Wereda Gizat and Mislene Gizat. The king has put administrative issues as the reason behind the adjustment of regions in that time. Here again Ethiopia was divided in 12 Awraja Gizats having 80 different Woreda Gizat and 334 Mislene Gizats. Wolaytta in this time was one of the 80 Woreda Gizats. Despite the rationale put forward by the king, Ethiopians in general and the people of Wolaytta in particular enjoyed nothing better even this time round [11].

Despite the changes in governments and a shift in ideology, Wolaytta's quest for statehood was sustained even during Derg period. It is obvious that people were killed for any reason standing against the centre where everything was decided in accordance with and interest of the socialist ideology. Here, the voice of the people was not as audible as the people are intended to shout.

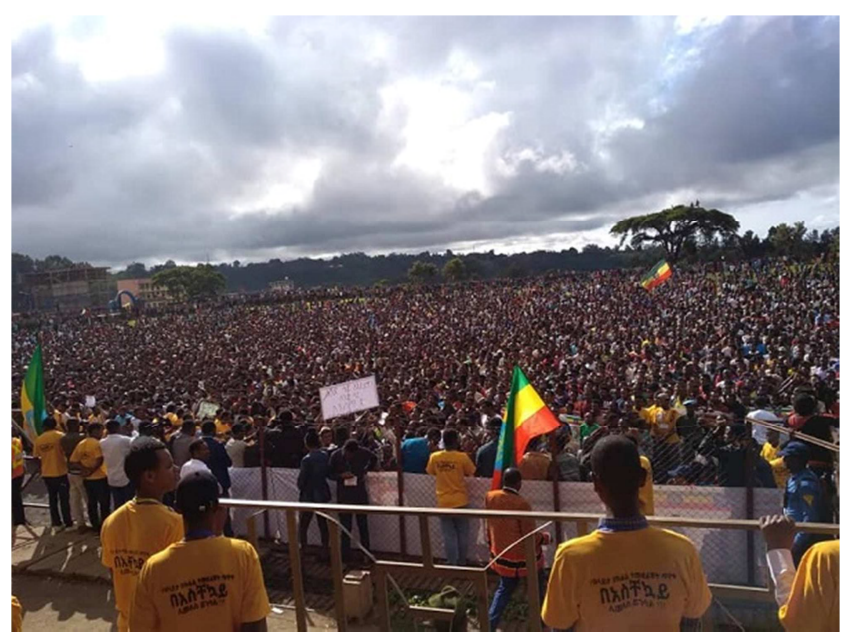

Figure 2. Hundreds of thousands of ethnic Wolyatta marched the streets of Wolayta Sodo in May 2019.

The quest for establishing statehood was never been as bold and organized as it was since the coming to power of the current regime. Efforts have been made to make Wolaytta and it's surrounding as one of the 14 states that was initially proposed in early 1990's. However, rulers of the period opted to neglect the quest and exclude Wolaytta from those that are proposed to be states during the early days of the current regime. The move however triggered pubic backlashes in major towns of Wolaytta. Before the revolt reaches to the climax, the state has managed to supress it.

And now with the imminent disintegration of the so called SNNPR, a superficial region designed for imminent extinction, Wolaytta demand of self-administration become the contemporary dialogue of the people. The extent of peoples' organization towards their demand, the imminent disintegration of the region and the loading changes in the political sphere in the Ethiopia makes the demand of Wolaytta people so different from that of the earlier one. 


\section{Conclusion}

Wolaytta is one of the strong nations that were led by its own dynasties having more than 50 kings. The history of this people dates back to the pre-history period reaching 56 thousand of years. They had their own currency by the name Merchuwa and managed to conquer northern Ethiopia up until Damot, northern Ethiopia. However, after Minilik invasion, Wolaytta lost all its hegemony and forced to live under poverty for almost one and half century. Still today, the socio economic and political problems of Wolaytta have been given a deaf ear. On top of that, they are forced to be administered under a superficial state that has never been in history and created only due to the interest of politicians.

Now the people of Wolaytta started massive movement that demand the highest possible administrative structure, a regional state. The demand is to have the ultimate political power to bargain with the central government and protect the interest of Wolaytta people who are forgotten by the federation. The movement seems to have the support of the mass especially the youths by the name Yalaga. The Yalaga successfully managed peaceful demonstration in Wolaytta region recently. The rally seems to continue up until the demand of Wolaytta people is answered.

Generally, Wolaytta's quest seems logical and appropriate taking the way regions are formed in Ethiopia. This people have history, enough territory, and population that make it to be one of the top 5 in terms of population size. Moreover, the current constitution of Ethiopia fully supports the demand as it is clearly mentioned inside this paper.

\section{References}

[1] Human Rights Council-HRCO (2017). 142nd Special Report: Human Right Violations Committed During the State of Emergency in Ethiopia. May 28, 2017, Addis Ababa.

[2] Brandt, S. A., et al. (2012). Early MIS 3 occupation of Mochena Borago Rock Shelter, Southwest Ethiopian
Highlands: Implications for Late Pleistocene archaeology, paleo-environments and modern human dispersals. Journal of Quaternary International 03: 047.

[3] Chiatti, R. (1984). The Politics of Divine Kingship in Wolaita (Ethiopia) 19th and 20th Centuries. PhD Thesis. University of Pennsylvania: 327-34.

[4] Berhanu, Bibiso (1995). Production practices in Damotta Woyde: A case of Sura Koyo. MA Thesis in Social Anthropology, Addis Ababa University.

[5] Adane Ayza (2016). Ye Gebar Sirat Be Wolaita:. 1887-1967 (In Amharic). Wolaita Sodo, p. 30.

[6] Demise Mojja (2009). A Survey of Socio-Political History of Wolaita to 1974. M. A. Thesis, Department of History, Addis Ababa University: 38-39, 55-56.

[7] Bahiru Zewde (2002). The Modern History of Ethiopia from 1885 to 1974, Addis Ababa University Press, Addis Ababa.

[8] Asela Gujubo (2012). Qedemt Wolaita and Kaffa (The Early Wolaita and Keffa), Addis Ababa: 34-68.

[9] Abreham Babanto (1979). The Origin of Lemlemitua Wolaitta and revolutionary Modern Wolaitta (In Amharic). Addis Ababa: United Printers.

[10] Federal Democratic Republic of Ethiopia Constitution (2005). Addis Ababa, Ethiopia.

[11] Fancho and Eyob (2014) Ye Wolaita Hizeb TarikEske 1966 (In Amharic Language). Wolaita Sodo, 2014.

[12] Jose'phine Lesur, Jean-Denis Vigne and Xavier Gutherz (2007) Exploitation of wild mammals in South-west Ethiopia during the Holocene (4000 BC- $500 \mathrm{AD})$ : the finds from Moche Borago shelter (Wolayta). Journal of Environmental Archaeology vol. 12 No. 2.

[13] Data Dea (2000). Clan, Kingdoms, and "Cultural Diversity" in Southern Ethiopia, Max Planck Institute for Social Anthropology, Germany: 163-188.

[14] Wana Wagesho (2004). Ye Wolaita Hizeb Tarik 2nd Edition (In Amharic), Addis Ababa: 14-23. 